\title{
The interplay between policy guidelines and local dynamics in shaping the scope of networks: the experience of the Italian Departments of Mental Health
}

\author{
Amelia Compagni*, Simone Gerzeli† and Mara Bergamaschi* \\ ${ }^{*}$ Centre for Research in Health and Social Care Management (CERGAS), Department of Institutional Analysis and Public \\ Management, Bocconi University, Milan; 'Department of Applied Statistics and Economics 'Libero Lenti', University of Pavia, \\ Pavia; ${ }^{\ddagger}$ Department of Management, University of Bergamo, Bergamo, Italy \\ E-mail: amelia.compagni@unibocconi.it
}

\begin{abstract}
Summary
In the mental health field, the creation of networks that can guarantee the smooth coordination of services and organizations across sectors is a priority in the policy agenda of several countries. In Italy, Departments of Mental Health (DMHs) have been designated responsible for the system of specialist mental health services, and also mandated as the conveners and leaders of interorganizational and cross-sectoral networks, by a system-wide reform. This study aims to understand how mental health networks have been assembled in this context and the factors and motivations that have shaped their scope. By combining an analysis of policies with a survey of DMH directors, we have determined that DMHs have preferentially formed collaborative relationships with social service providers (local governments) and the voluntary sector. In contrast, relationships with substance abuse and addiction services and primary care providers were weak and stifled by a lack of trust and by conflict about respective contributions to mental care. We explore the reasons for this selectivity in interorganizational relationships and propose that a lack of targeted incentives in policy guidelines, on the one hand, and the existence of a mandated network leadership, on the other, have led to a rather narrow range of collaborations.
\end{abstract}

\section{Introduction}

Since the 1990s, the creation of networks among organizations that provide public services, in particular in the welfare and health sectors, has been at the centre of the political programmes of several countries.

The provision of mental health $(\mathrm{MH})$ services is an ideal example. It is thought that an effective approach to mental ill health must offer patients housing support, employment, peer-to-peer counselling and social inclusion initiatives, all in addition to health care services. Therefore, the MH policies of most countries advocate collaboration within the $\mathrm{MH}$ sector, as well as across welfare

Amelia Compagni PhD, Assistant Professor, Centre for Research in Health and Social Care Management (CERGAS), Department of Institutional Analysis and Public Management, Bocconi University, Milan, Italy.

Simone Gerzeli MSc, Researcher, Department of Applied Statistics and Economics 'Libero Lenti', University of Pavia, Pavia, Italy.

Mara Bergamaschi MSc, Researcher, Department of Management, University of Bergamo, Bergamo, Italy. sectors, as the primary method to guarantee continuity of care and an effective level of integration across services. ${ }^{1,2}$ However, how to achieve effective and comprehensive $\mathrm{MH}$ networks, and the role of policy in this, is still debated at an international level.

$\mathrm{MH}$ networks have been described thoroughly and it is well understood how the range and diversity of their contributors are shaped by the relationships between these organizations. ${ }^{3-7}$ For example, studies have identified several reasons for the initiation of collaborations across service organizations: (1) resource-based theories emphasize that, in a situation of scarcity, organizations collaborate in order to access resources that would be otherwise unavailable; (2) institutional-based theories suggest that professional norms shape collaborations; and (3) exchange theories indicate that collaborations are need-oriented and that they are started because they allow agencies to reach otherwise attainable objectives. ${ }^{8-10}$

In contrast, the extent to which the final scope of $\mathrm{MH}$ networks is influenced by the interplay between local dynamics among partners and the directions of policy-makers has yet to be explored. It is of particular importance to address this issue when networks are 
promoted through system-wide reforms at the national level, with policy-makers often unaware of the factors that might facilitate or impede effective implementation at the local level.

In the present study, we focused on two aspects that are thought to be critical in shaping a network: (1) the system of incentives that promote the initiation of certain relationships and (2) the model of governance of the network. We addressed the following questions: Should policy-makers design incentives that will promote the formation of certain networks and should they determine in advance who will lead a network or should this be left to the action and autonomy of professionals and managers and be determined by local dynamics? What are the consequences of such choices?

Previous studies have shown that policy-makers have been keen to present networks as driven, almost mechanistically, by a common and unifying goal and reticent to design specific incentives or mechanisms to steer them actively. A notable exception was the implementation of pooled budgets in England and Sweden, where health and social care organizations were forced to share resources for the purpose of providing $\mathrm{MH}$ services jointly. ${ }^{11} \mathrm{~A}$ second example is provided by joint commissioning in England, where the health and social sectors were asked to agree on the range of $\mathrm{MH}$ services to be planned for a community. ${ }^{12}$ However, neither approach was considered to be entirely successful.

Policy-makers also have shown a controversial attitude with respect to the governance of networks. The notion that networks should be governed and controlled has been neglected for a long time. ${ }^{13}$ In contrast to more traditional, hierarchy-based models, networks have been thought of as self-assembling entities in which governance is shared among partners, and as such, they are not subject to active control. ${ }^{10,14,15}$ In addition, it remains uncertain which models of network governance should be promoted, and studies of the most effective solutions are lacking. ${ }^{10}$ For instance, in the specific case of $\mathrm{MH}$, there is a longstanding debate about whether a centralized $\mathrm{MH}$ agency that manages the network or a decentralized network of agencies that are coordinated around the issue of $\mathrm{MH}$ is preferable. ${ }^{3,16-19}$ Some countries, such as the USA, have experimented with creating single $\mathrm{MH}$ authorities to work as 'systems case managers'. ${ }^{19}$ Similarly, in the 1990s, England was organized into MH Trusts and, later, Health and Social Care Trusts in which MH service providers from different sectors were united within the same organizational entity and charged with working jointly. Some authors have concluded that a highly centralized $\mathrm{MH}$ network broker does not necessarily guarantee more effective care but at least improves integration among the participating organizations. ${ }^{18}$ However, the debate about network governance is ongoing and any choices made at policy level will be affected by the high degree of uncertainty about its effects.

The Italian MH system is an ideal subject for an analysis of the role played by policy in shaping the scope of networks. In 1978, a national law ruled that standalone psychiatric hospitals were to be closed progressively and replaced by a new range of community-based $\mathrm{MH}$ services. ${ }^{20}$ In the mid-1990s, as a result of the first National Mental Health Plan, a nationwide reform led to the ex novo creation of Departments of Mental Health (DMHs) across the whole national territory, each being responsible for all specialist $\mathrm{MH}$ services in a defined catchment area. ${ }^{\text {a21 }}$ Most importantly for the aim of this study, policymakers charged $\mathrm{DMHs}$ with not only organizing all specialist $\mathrm{MH}$ services but also convening and leading interorganizational and cross-sectoral networks in the area of $\mathrm{MH}$.

The aim of the present study was to investigate which elements within policy guidelines might affect the final scope of $\mathrm{MH}$ networks through interplay with local dynamics. The paper is organized as follows. Firstly, we analyse the content of the Italian $\mathrm{MH}$ reform and its underlying policies with respect to the creation of networks; we pay particular attention to how MH networks were envisaged by policy-makers, what incentives were put into place to achieve their creation and what role was attributed to $\mathrm{DMH}$ in the governance of $\mathrm{MH}$ networks. Secondly, we describe the breadth of the collaborations established by DMHs. In the discussion, we offer several explanations for the rather narrow scope of the $\mathrm{MH}$ networks that $\mathrm{DMH}$ s have created.

\section{Methodology}

We chose to adopt two different and sequential approaches.

\section{Analysis of policies for the reform of Italian $\mathrm{MH}$ services}

For the period of 1980-2008, policy documents at national and regional levels were collected from the databases of the Ministry of Health, regional governments and the National Agency on Regional Healthcare Systems. The most relevant national policy documents (National Mental Health Plans, 1994-1996 and 1998-2000, respectively) and more than 200 regional documents were analysed. Texts were coded by two researchers on the basis of the following keywords: 'continuity', 'integration', 'integrated', 'coordination', 'network', 'unity', 'relationship', 'linkage' and 'collaboration'. The following categories were retrieved: (1) motivations given for the creation of MH networks; (2) the role of the DMH and its director; (3) the composition of the network; and (4) solutions to promote the formation of networks. The interpretation of the policy documents was confirmed by interviews (6 in total) with four DMH directors and two

\footnotetext{
${ }^{\mathrm{a}}$ The 205 current DMHs must offer four types of care: acute care in the psychiatric ward of a general hospital, community-based care through community $\mathrm{MH}$ centres and ambulatories, semi-residential care and residential care. DMHs fall under the jurisdiction of the respective local health authorities (LHAs), which are in charge of the overall health of a certain territory. Each DMH is allocated a budget by its LHA and is managed by a department director and a DMH Board.
} 
experts who had participated in the formulation of the national MH reform agenda during the 1990s. Analysis of the documents also enabled the identification of organizations and stakeholders that were supposed to be part of the $\mathrm{MH}$ networks and whose relationships with DMHs could then be investigated through the survey.

\section{Survey \\ Description of the sample and study limitations}

The target population of the survey consisted of all 205 directors of the DMHs. There is no public database of Italian DMHs. Addresses were compiled from the websites of the individual DMHs and the questionnaire was sent by post to the corresponding DMH directors. Two rounds of telephone recalls (after 3 and 6 weeks) were conducted. In total, 150 out of $205 \mathrm{DMH}$ directors were reached by mail (coverage rate 73\%) and, of these, 53 returned the questionnaire (return rate $35 \%$ ).

The modest survey response rate is the main limitation of this work, since it is not possible to exclude some bias among respondents. For instance, it cannot be ruled out that the directors who responded to the survey were more prone to interact with other stakeholders in $\mathrm{MH}$ networks than those who did not respond. In addition, it is difficult to assess how well these $53 \mathrm{DMHs}$ represent the situation in Italy overall. Despite this, some factors make us reasonably confident that, although the study shows only a partial picture, it gives a first indication of how $\mathrm{MH}$ networks have been shaped within Italy. Firstly, the average DMH catchment area in the sample consisted of 350,000 inhabitants, which corresponds to the average size of an Italian LHA, and indicates that the sample was distributed around the mean for Italy (Table 1). Secondly, the DMHs that responded were distributed uniformly across the national territory, although departments in Northern Italy were represented to a greater extent in the sample than those in Central-Southern Italy (Table 1). In addition, the DMHs in the sample served a reasonably large proportion of the total Italian adult population $(40 \%)$.

Table 1 Main characteristics of the DMHs under investigation

\begin{tabular}{lll}
\hline $\begin{array}{l}\text { Geographical distribution } \\
\text { Northern Italy }\end{array}$ & Total DMHs & Sample \\
Central-southern Italy & $50 \%(102 / 205)$ & $62 \%(33 / 53)$ \\
Total & $50 \%(103 / 205)$ & $38 \%(20 / 53)$ \\
Variables & 20553 & \\
& $\begin{array}{l}\text { Average per DMH } \\
\text { (min-max) }\end{array}$ \\
Population in catchment area & 350,000 \\
& $(50,000-1,400,000)$ \\
Number of community-based & $7.0(1-31)$ \\
centres & $3.6(1-26)$ \\
Number of hospital wards & $4.6(1-14)$ \\
Number of semi-residential units & $11.0(1-44)$ \\
Number of residential units & $27.0(6-60)$ \\
Total number of care units & $147.0(8-597)$ \\
Number of DMH employees &
\end{tabular}

DMH, Department of Mental Health
Overall, the DMHs in the sample showed a high level of variability, in relation to both their size and number of employees, as well as the number of units for each of the four types of care that a DMH must provide (Table 1). This is unsurprising because the directors of DMHs, while respecting the national $\mathrm{MH}$ framework and its service requirements, have the autonomy to organize their departments as they deem appropriate. Despite this high variability, the analysis revealed a clear trend in the behaviour of the DMHs with respect to the formation of networks and enabled some general, although still preliminary, conclusions to be drawn for the Italian MH system overall.

\section{Questionnaire structure}

The questionnaire was composed mainly of closed questions but a few open questions allowed the DMH directors to comment on the barriers that they perceived to exist to the establishment of some collaborations, the level of commitment of other partners to the creation of networks and the benefits that were gained from collaboration. Most directors were also interviewed to clarify their answers further. The questionnaire was validated twice by a group of $\mathrm{MH}$ experts and $\mathrm{DMH}$ directors and comprised 35 questions with the following sections:

(1) Information about the DMH director (education, time in role, time spent in clinical activity);

(2) Characteristics and complexity of the DMH (catchment area, location, number of units per care type, private accredited providers in the same catchment area, number of employees);

(3) Coordination within the intraorganizational network, in which two levels were distinguished: (a) coordination among DMH units and (b) coordination with other specialist MH service providers, such as private accredited providers of residential care and child and adolescent MH services (CAMHS);

(4) Coordination with other organizations and stakeholders involved in mental care (interorganizational network). The partners examined were: (a) local governments (LGs), which are responsible for social services; (b) the third sector (e.g. patients' and families' associations, social cooperatives); (c) general practitioners (GPs) and primary care; (d) substance abuse and addiction services (SAAS).

\section{Index creation}

To avoid asking DMH directors for their perception of the extent of intra- and interorganizational networks, each concept was operationalized with respect to a few elements and to reconstruct the degree of coordination, interaction was considered at two levels, service and management, as suggested by the literature. ${ }^{22}$ Whereas, at the service level, specific tools by which coordination could be achieved were taken into consideration, at the management level, the frequency of communication and meetings among organizations or units was used as an indicator of the degree of collaboration. ${ }^{23-25}$ 
For the intraorganizational network, the coordination of services was operationalized with respect to three aspects: (1) the frequency of joint case review among professionals that belonged to different DMH units; (2) the degree of sharing of clinical information through a common information technology (IT) system; and (3) the creation of care pathways across DMH units (Table 2). The involvement of private accredited $\mathrm{MH}$ providers in these aspects was investigated. Regarding the relationship between the DMH and CAMHS, the two factors taken into consideration were collaborative agreements for joint case review and the creation of protocols for the referral of CAMHS users to the DMH when of age.

With respect to interaction at the management level, questions addressed the degree of participation of the different DMH units in the management of the department overall and the organization of services across units. An understanding of the governance structure of the DMHs and the composition of DMH boards was obtained by the analysis of documents. The involvement of private accredited providers and CAMHS were included in this level of analysis. Concerning the interorganizational network, the degree of coordination of services was

Table 2 Tools to assess service coordination in intra- and interorganizational $\mathrm{MH}$ networks

\section{Intraorganizational networks}

- Frequency of joint case review among professionals who belong to different $\mathrm{DMH}$ units

- Degree of clinical information sharing through a common IT system

- Creation of care pathways across DMH units

Interorganizational networks

$\mathrm{DMH} \leftrightarrow$ local government

- Frequency of client referral and sharing of clinical information

- Creation of joint services and initiatives

- Presence of mixed care teams

$\mathrm{DMH} \leftrightarrow$ third sector

- Existence of a list of all services offered by the third sector and its availability to users and families

- Inclusion of services provided by the third sector in the therapeutic protocols and care pathways elaborated by the $\mathrm{DMH}$

- Creation of joint services and projects

$\mathrm{DMH} \leftrightarrow \mathrm{GPs}$ and primary care

- Existence of liaison services for GPs to support the diagnosis of early signs of mental illness and the management of mild mental health problems

- Agreements with GPs for the joint care of users with serious mental illness who frequently present physical comorbidities in need of continuous monitoring by a GP

- Training or education programmes about mental health for GPs

DMH $\leftrightarrow$ substance abuse and addiction services (SAAS)

- Elaboration of treatment protocols and care pathways across the two organizations for consumers with a dual diagnosis

- Sharing of clinical information about users treated jointly by the DMH and SAAS

- Presence of DMH personnel in detoxification communities staffed normally by SAAS professionals

$\mathrm{MH}$, mental health; DMH, Department of Mental Health; IT,

information technology; GP, general practitioner reconstructed by enquiring about the establishment of three types of tool for interaction between the DMH and each of the counterparts. The choice of tools was based on the literature and tailored according to the partner as appropriate (Table 2). A concise index, which was labelled 'service coordination', was established by applying the following rule: if the DMH had put in place either one or none of the three tools, it was considered to have a 'low level of service coordination', whereas if the DMH had put in place two or three of the tools for coordination proposed by the survey, it was considered to have a 'high level of service coordination'.

With respect to interaction at the management level, three aspects (frequency of meetings, topics of discussion, participation of DMH middle management) were considered important in determining the nature of the relationship between a DMH and the other potential members of an $\mathrm{MH}$ network. Only the frequency of meetings was used to build a concise index, which was labelled 'management interaction'. Answers such as 'never', 'once a year' and 'delegated to others' were classified as 'low management interaction', whereas 'several times a year' and 'every month' as 'high management interaction'. By combining the two indexes ('service coordination' and 'management interaction') and their intensities (low and high), four categories of classification were obtained: (1) low service coordination and management interaction; (2) high service coordination and management interaction; (3) low service coordination and high management interaction; and (4) high service coordination and low management interaction.

\section{Findings}

\section{Policy guidelines: the desired structure of $\mathrm{MH}$ networks}

In Italy, integration, coordination and interorganizational relationships are recurrent themes in national and regional policy documents that are related to $\mathrm{MH}$. The two National Mental Health Plans, which marked the creation of DMHs and are still the most relevant policy documents in this domain, stated explicitly that the DMH was a network in itself and had the organizational authority to bring together and coordinate the range of specialist $\mathrm{MH}$ services in a certain catchment area. DMHs were established as 'the coordinating bodies in charge of guaranteeing unity and integration of the whole network of psychiatric services in one territory'. The main motivation given for the creation of DMHs in the national reform of the Italian $\mathrm{MH}$ system was that the shift from psychiatric hospitals to community-based services had generated a plurality of stakeholders involved in mental care provision, and resulted in redundancy, high levels of complexity and the fragmentation of services. The creation of a single organizational entity, the DMH and homogeneously across the whole national territory was proposed as the only means to rationalize this new $\mathrm{MH}$ system and, indeed, the DMH became its core module. 
In addition to guaranteeing the integration and continuity of care across specialist mental health care services, DMHs were charged by policy-makers with the responsibility to 'engage and link with all social services and other agencies that operate at the boundary with mental health'. The DMH was mandated as the 'sector coordinator' and designated as the lead organization, the convener of the proposed $\mathrm{MH}$ network. The job description of the Department Head, as the 'lead coordinator', was mainly to 'guarantee continuity of care and coordination among organizations'.

Policy guidelines stated clearly the stakeholders part of the $\mathrm{MH}$ system with whom the DMH was supposed to create links and collaborate: CAMHS, within the $\mathrm{MH}$ sector itself; primary care (GPs and providers of maternal and child health services), SAAS, social services and the third sector (voluntary associations, social cooperatives, consumer groups), within the broader system of stakeholders involved in $\mathrm{MH}$. Some contributors were singled out as preferred collaborators. For example, the Second National Plan advocated strongly the creation of links with CAMHS as well as with schools, paediatricians and hospital paediatric departments. Local Governments (LGs), which are responsible for social services, were also presented as natural collaborators for DMHs in both the planning of services and the provision of housing and employment opportunities to patients of the $\mathrm{DMH}$. Finally, the third sector was often acknowledged as an important local resource.

Regional policies, which assumed a greater relevance in the 1990s after the progressive devolution of power to the regions, reiterated these concepts further. They also often distinguished between a formal network (GPs, LGs, other health and social services, hospital departments, etc.) and an informal or non-statutory network and reaffirmed the need for the DMH not only to assemble these networks but also to govern them.

Despite this strong mandate, the need for integration and collaboration across organizations has been poorly justified in policy documents. It was argued that coordination was necessary to achieve 'real effectiveness' of services for people with $\mathrm{MH}$ problems; the opportunity to take advantage of all resources (i.e. services, workforce and funding) in a certain territory and 'system optimization' were presented as the main advantages of working in a network. In addition, the degree of integration that should have been pursued by the different contributors involved in mental care was not described, and the incentives and tools available to achieve $\mathrm{MH}$ networks were not detailed. The only strategies that were proposed for the creation of $\mathrm{MH}$ networks were shared care plans for each patient, the creation of a 'pact for mental health' stipulated by all contributors and a series of guidelines that regulated the relationships between the $\mathrm{DMH}$ and all other stakeholders.

In conclusion, through a system-wide reform of the MH system in Italy, policy-makers have established the prime mission of the DMHs to be the creation of intraorganizational networks among DMH units and other providers of specialist $\mathrm{MH}$ services, and of interorganizational networks with stakeholders in the health and social sectors (Figure 1).

\section{Intraorganizational relationships: how DMHs interpret their identity as $\mathrm{MH}$ networks}

We examined coordination at the level of both service and management, firstly among DMH units and, then, between the DMH and other specialist MH service providers, such as private accredited residential facilities and CAMHS.

The analysis showed that DMHs have developed different degrees of internal service coordination. In the sample, three main groups could be distinguished. Approximately $10 \%$ of the DMHs did not have an IT system that enabled the sharing of clinical data across the $\mathrm{DMH}$, conducted joint case reviews only rarely, or very rarely, and had not developed any care pathways. None of these DMHs involved professionals from private accredited facilities in clinical case reviews and none had established formal relationships with CAMHS (low intraorganizational network, Table 3). A second group, which comprised a third of the DMHs, relied mainly on joint case reviews and the sharing of clinical data as the means of coordination and working together, but had not developed any care pathways (medium intraorganizational network). Most of these DMHs neglected their connections with private accredited facilities and professionals: only $37 \%$ included them in case reviews and approximately half of the DMHs shared clinical information with them through a common IT system (Table 3). Finally, the third group within the sample performed joint case reviews and shared clinical data but had also established care pathways elaborated by multidisciplinary teams that belonged to different $\mathrm{DMH}$ units (high intraorganizational network). Professionals from private facilities were included rarely in the process of elaborating care pathways (Table 3 ).

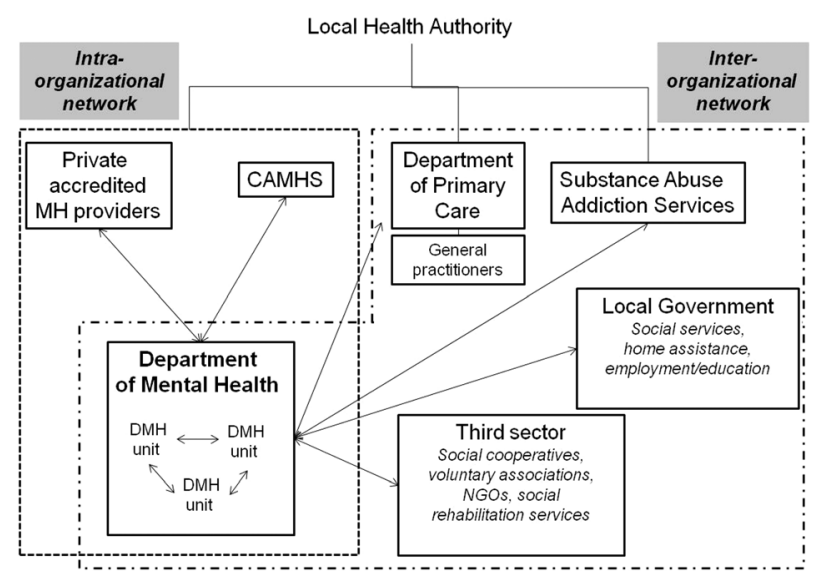

Figure 1 Schematic representation of the intra- and interorganizational networks that DMHs were charged with creating. Accountability lines are indicated within the Local Health Authority. DMH, Department of Mental Health; CAMHS: children and adolescent mental health services 
Table 3 Characterization of respondent DMHs according to the degree of interaction among DMH units and with other specialist MH service providers

\begin{tabular}{|c|c|c|c|c|c|c|}
\hline \multirow[b]{2}{*}{$\begin{array}{l}\text { Degree of } \\
\text { intraorg. network }\end{array}$} & \multirow[b]{2}{*}{$\begin{array}{l}\text { DMHs (\% of } \\
\text { sample) }\end{array}$} & \multicolumn{2}{|l|}{ Among DMH units } & \multicolumn{2}{|c|}{$\mathrm{DMH} \leftrightarrow$ private $\mathrm{MH}$ providers } & \multirow{2}{*}{$\begin{array}{l}\mathrm{DMH} \leftrightarrow \mathrm{CAMHS} \\
\begin{array}{l}\text { Collaboration } \\
\text { protocols }\end{array}\end{array}$} \\
\hline & & $\begin{array}{l}\text { Frequent joint case } \\
\text { revision and/or IT system }\end{array}$ & $\begin{array}{l}\text { Care } \\
\text { pathways }\end{array}$ & $\begin{array}{l}\text { Involvement in case } \\
\text { review }\end{array}$ & $\begin{array}{l}\text { Involvement in data } \\
\text { sharing }\end{array}$ & \\
\hline Low & 10 & - & - & - & - & - \\
\hline Medium & 36 & $\sqrt{ }$ & - & $37 \%$ & $47 \%$ & $53 \%$ \\
\hline High & 54 & $\sqrt{ }$ & $\sqrt{ }$ & $36 \%$ & $46 \%$ & $71 \%$ \\
\hline
\end{tabular}

CAMHS, child and adolescent mental health services; $\mathrm{MH}$, mental health; DMH, Department of Mental Health; IT, information technology

The relationships of the DMHs with CAMHS were more intense and formalized than those with private accredited facilities: a substantial proportion had stipulated collaboration protocols with CAMHS, mostly for the correct referral of patients to the DMH once they became of age and less frequently for joint case review (Table 3). We could not observe any relationship between the level of complexity of the DMH (size, number of units and employees) and the degree of intraorganizational networking.

With respect to the degree of coordination at the management level within the $\mathrm{DMH}$ and with other $\mathrm{MH}$ service providers, all DMH directors met several times a year with the heads of DMH units to discuss clinical and organizational issues. From the available documents, we concluded that the director is held primarily accountable for the performance and management of the DMH whereas the DMH boards have, for the most, a supporting role. The composition of the boards reflected the multidisciplinary nature of the DMH workforce, in a structure that is dominated otherwise, at the top and middle management levels, by psychiatrists. Meetings between the $\mathrm{DMH}$ director and unit heads were the preferred way of establishing connections among DMH units, rather than meetings with the DMH board. Private providers were regularly not included in this loop whereas there was a degree of functional interaction with directors of CAMHS units.

\section{Interorganizational relationships: how DMHs interpret working in $\mathrm{MH}$ networks$$
\mathrm{DMH} \leftrightarrow \mathrm{LG}
$$

The great majority $(81 \%)$ of DMHs stated that they had created joint services together with LGs. These mainly took the form of mixed teams for home visits for people with mental problems and for the elderly in nursing homes (which are owned mostly by LGs). LGs were also instrumental in finding job placements and housing for patients of the $\mathrm{DMH}$, often within voluntary $\mathrm{MH}$ associations and social cooperatives.

Communication among professionals who belong to the two organizations and client exchanges appeared intense. For example, $90 \%$ of the respondents indicated that social workers employed by the LG contacted the DMH often or very often, to deal jointly with serious cases such as socially isolated and aggressive individuals and multiproblem families.

Similarly, interaction at the management level was depicted as lively and relevant (Table 4). Among the DMH directors, $65 \%$ met several times a year or every month with the head of social services of the LG. Only $8 \%$ of the DMH directors never met with these heads. Exchanges were mainly about common projects such as the planning of joint services and the allocation of human and economic resources to shared initiatives. The participation of DMH middle management in these meetings was also quite high: $92 \%$ of directors indicated that unit heads participated regularly (Table 4). This suggests that the relationship with LG is considered relevant across the department and is more interorganizational than interpersonal in nature.

Overall, the distribution of DMHs was skewed towards a high degree of collaboration and interaction with LGs, at both the service and management levels, with approximately $60 \%$ of the $\mathrm{DMHs}$ in this category (Figure 2). The fact that $21 \%$ of DMHs showed high service coordination and low management interaction, might indicate the existence of a strong collaboration that does not require frequent reinforcement by top management.

Table 4 Interorganizational relationships at managerial level: frequency of meetings and degree of participation by $\mathrm{DMH}$ middle management

\begin{tabular}{cccc}
\hline & \multicolumn{3}{c}{ \% of sample } \\
\cline { 2 - 4 } & $\begin{array}{l}\text { Never, once a } \\
\text { year, delegated } \\
\text { to others } \\
\text { (never) }\end{array}$ & $\begin{array}{l}\text { Several } \\
\text { times a } \\
\text { year, every } \\
\text { month }\end{array}$ & $\begin{array}{l}\text { No participation } \\
\text { by DMH middle } \\
\text { management }\end{array}$ \\
\hline $\begin{array}{c}\text { DMH } \leftrightarrow \text { local } \\
\text { governments }\end{array}$ & $34.6(8 \%)$ & 65.4 & 8.3 \\
$\begin{array}{c}\text { DMH } \leftrightarrow \text { third } \\
\text { sector }\end{array}$ & $7.7(2 \%)$ & 92.3 & 6.1 \\
$\begin{array}{c}\text { DMH } \leftrightarrow \text { GPs } \\
\text { and primary } \\
\text { care }\end{array}$ & $34.6(25 \%)$ & 65.4 & 31.4 \\
DMH $\leftrightarrow$ SAAS & $43.1(18 \%)$ & 56.9 & 29.4 \\
\hline
\end{tabular}

SAAS, substance abuse and addiction services; $\mathrm{MH}$, mental health; DMH, Department of Mental Health 


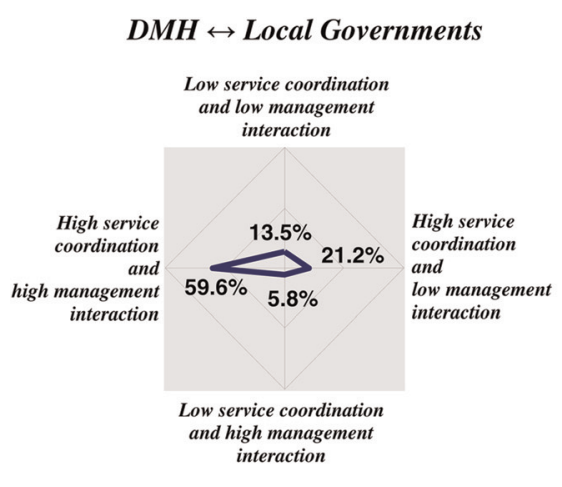

$D M H \leftrightarrow G P S$

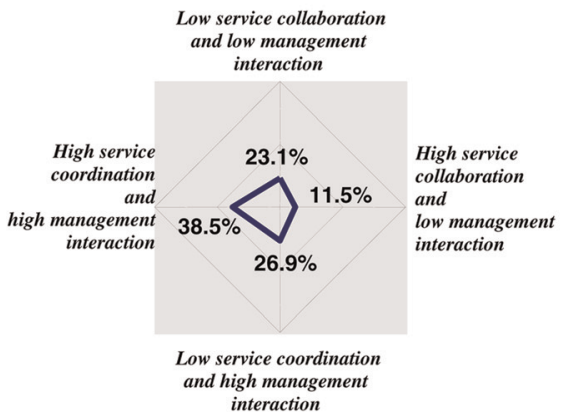

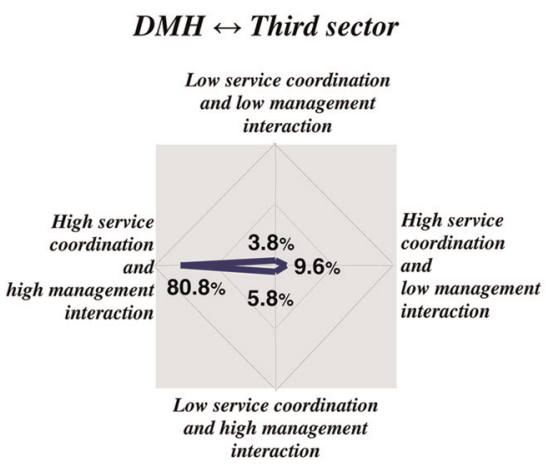

$D M H \leftrightarrow S A A S$

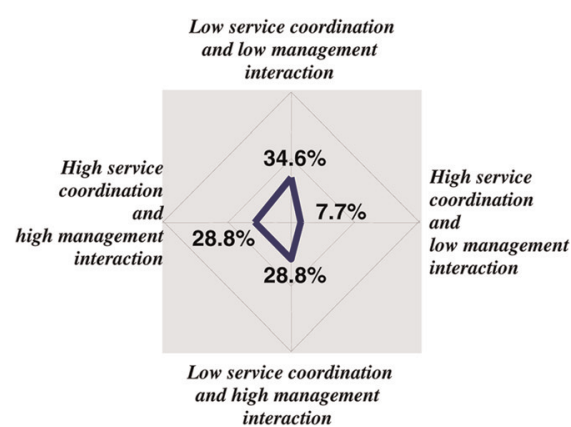

Figure 2 Distribution of DMHs according to their relationships with network partners. Percentages indicate the proportion of $\mathrm{DMHs}$ in the four respective categories of high/low service coordination and high/low management interaction. DMH, Department of Mental Health

\section{$\mathrm{DMH} \leftrightarrow$ third sector}

The intensity of the interaction between DMHs and the non-profit or third sector, at both service and management levels, appeared even stronger than that between DMHs and LGs. In general, the majority of DMHs (86.5\%) had a list of services offered by the third sector and made it available to patients and families. These same services were included in the care pathways and protocols of the DMH. Joint services were focused mainly on social rehabilitation and the employment/training of users. Through the survey, we also understood that services provided by the third sector were sometimes located within the $\mathrm{DMH}$, with some DMH personnel seconded to these organizations. At the same time, volunteers were utilized by the $\mathrm{DMH}$ and their services financed by the $\mathrm{DMH}$ itself. This made the exchange between DMHs and the third sector very intense but also indicated the financial reliance of non-profit organizations on DMHs. In addition, $70 \%$ of the respondents stated that voluntary associations, consumers and families were represented on the $\mathrm{DMH}$ board.

At the management level, meetings between the $\mathrm{DMH}$ directors and the heads of non-profit associations and social cooperatives occurred frequently (Table 4): $20 \%$ of the respondents met with these partners every month and only very few (4.4\%) did not involve DMH middle management in these meetings. Discussions focused on the evaluation of current projects, on the planning of new ones, and more rarely on the allocation of economic resources. A third of DMH directors had instituted an internal office to deal with the external relationships of the department and normally these offices focused on relationships with the third sector. In summary, the intensity of interaction between DMHs and the third sector was strong, with $81 \%$ of the departments showing a high degree of collaboration at both service and management levels and only $4 \%$ belonging to the category of low service coordination and management interaction (Figure 2).

\section{$\mathrm{DMH} \leftrightarrow \mathrm{GPs}$ and primary care}

The survey revealed that only 33\% of the DMHs had formulated specific agreements with GPs for the joint care of patients with serious mental illness. When agreements were in place, approximately half of the GPs in a catchment area participated in the programme and conducted joint case reviews with professionals from the DMH. The creation of a liaison service appeared much more common $(66 \%)$, and, when in place, was used by approximately $40 \%$ of the GPs in a catchment area. Finally, $40 \%$ of the DMHs in the sample offered education programmes in $\mathrm{MH}$ issues to GPs, even if sporadically.

GPs are dispersed throughout the territory, hence engagement might be difficult. However, given the level of participation of GPs in the initiatives of DMHs, GPs appeared to be interested in these topics. Despite this, 
the DMH directors who responded expressed a rather negative view of this type of collaboration. Two problems were mentioned more frequently than others: the reticence of GPs to address $\mathrm{MH}$ issues and their lack of competence.

At the management level, the $\mathrm{DMH}$ director can be faced with different counterparts in terms of primary care providers and GPs. For simplicity, we refer here to the Department of Primary Care (DCP). In Italy, GPs are independent professionals who are paid on a capitation basis by the LHA. The top management of the DCP is a point of reference for GPs and for policies in primary care but has no direct responsibility for the activities of GPs. The frequency of meetings between the managers of DMHs and DCPs was quite high overall, although $25 \%$ of the DMH directors in the sample never met their counterpart in primary care (Table 4). The meetings between the DMH directors and heads of DCPs often focused on how to improve the competence of GPs in the provision of $\mathrm{MH}$ services. Participation by $\mathrm{DMH}$ middle management was substantially lower than in the interaction with LGs and the third sector (Table 4).

Overall, the relationship DMH $\leftrightarrow$ GPs was weaker than that with LGs and the third sector. Of the respondents, $50 \%$ showed high service coordination for GPs in comparison with $81 \%$ and $90 \%$ for LGs and the third sector, respectively, and $23 \%$ were in the category of low service coordination and management interaction (Figure 2).

\section{$\mathrm{DMH} \leftrightarrow$ substance abuse and addiction services}

The majority $(68 \%)$ of DMHs did not share diagnostic or evaluation protocols with SAAS. Half of the DMHs in the sample had elaborated care pathways together with SAAS for the treatment of users with dual diagnosis. Nearly all were able to share clinical information across the two organizations, with the most frequent mode being through informal personal relationships among professionals. The DMHs that had put in place several of these collaborative solutions, corresponded to those in which at least $50 \%$ of the users with dual diagnosis were cared for jointly with SAAS. This indicated that modes of collaboration could lead quite effectively to the sharing of patients. However, more than $60 \%$ of the DMHs appeared to have elaborated their own treatment and service protocols for dual diagnosis patients rather than relying on collaboration with SAAS.

Several remarks by DMH directors revealed that SAAS are perceived to be services that are weak, both in economic terms and with respect to workforce ('they do not have beds', 'they do not have psychiatrists'). The main difficulties in this relationship appeared to be the cultural differences between the two organizations and their divergent approaches to treatment and care. More than $60 \%$ of the DMHs showed a low degree of coordination with SAAS at the service level, as compared with 10$20 \%$ in the case of LGs and the third sector (Figure 2). In part, this was reflected at the management level where the frequency of meetings was lower than that with other contributors and $18 \%$ of $\mathrm{DMH}$ directors never met the corresponding head of SAAS (Table 4). The content of these meetings focused mainly on clinical cases who were cared for jointly by the two entities and rarely concerned organizational issues. In addition, participation by $\mathrm{DMH}$ middle managers was rather poor and approximately $30 \%$ of respondents stated that unit heads were never invited to these meetings.

\section{Discussion}

By combining the analysis of national and regional policies with a survey of DMHs, the study reported herein assessed the current state of $\mathrm{MH}$ networks in Italy, their scope and the nature of the relationships that characterize them, and compared this to how policy-makers had envisaged it to be in the national reform of the $\mathrm{MH}$ system. The study also attempted to explain the degree to which policy guidelines and local dynamics have been critical in shaping the final scope of $\mathrm{MH}$ networks.

In the first instance, DMHs were created to coordinate all specialist $\mathrm{MH}$ services. The analysis suggested that DMHs have partially reached this objective and that they relied on strategies that could be considered professional-centred (e.g. joint case reviews), rather than organizational or managerial (e.g. clinical pathways), to create internal networks among their own units. Interactions with other specialist $\mathrm{MH}$ providers, such as private residential facilities and CAMHS, which are just outside the boundary of the $\mathrm{DMH}$, are still neglected or formalized mostly for administrative reasons.

DMHs were also supposed to initiate and manage broad MH networks with a variety of stakeholders. Policy-makers did not state explicitly how this should be achieved, assumed fairly neutral pre-existing relationships and did not propose specific incentives to steer the network in the desired direction. The results of the study demonstrated that DMHs have developed, rather consistently, a narrow range of collaborations and have strengthened only certain relationships, in particular with LGs and the third sector.

The analysis suggests that local dynamics have been critical in leading DMHs to this selection of collaborations. Firstly, the services provided by LGs and the third sector are complementary and interdependent to those of DMHs because these organizations offer services namely, employment, housing, peer-to-peer counselling that DMHs are unable to provide on their own. Secondly, resources, such as funding, staff and competence, that are offered by LGs and the third sector appear tangible and accessible, a factor that has already been shown to facilitate the establishment of collaborative relationships. ${ }^{26}$ In the case of the third sector, DMHs have even 'incorporated' voluntary associations (including personnel and services) into their organizational boundaries. An additional element that might explain the narrow scope of the interorganizational relationships of DMHs is that LGs and the third sector are geared mainly to the needs of users with serious mental illness, and the core clients 
for whom DMHs were created. It is possible that DMHs might have made a deliberate choice to limit the $\mathrm{MH}$ network to these participants and not to engage others, such as GPs and SAAS, to avoid responsibility for a potentially large user population for which resources and competence were not available within the DMH. In the case of GPs and SAAS, relationships with DMHs appear weak at the level of both service and management. Professional dynamics, low levels of trust, and poor evaluation of the other's work and competence were driving factors in preventing the development of these relationships.

We propose that, in the context of very weak guidance by policy and the lack of incentives, local dynamics and organizational interests prevail in the formation of networks. These findings suggest that to build comprehensive $\mathrm{MH}$ networks it is necessary to devise incentives that promote, in a targeted and intentional manner, those relationships that are predicted to be weak. For instance, the outputs of certain collaborations could be rewarded. Mur-Veeman et al. ${ }^{27}$ recommend that a mixture of specific measures that are suitable for local circumstances and general measures, both financial and legislative, is critical to facilitate interorganizational collaboration. Policies that make explicit to organizations the goals to be achieved through collaboration as well as concrete tools to achieve them, might be just as instrumental.

We suggest that the narrow scope of $\mathrm{MH}$ networks observed in the Italian context also depended on the choices made at policy level concerning network governance. Policy-makers mandated DMHs as the leaders of $\mathrm{MH}$ networks, which is a common strategy in public sector networks. However, when the DMHs were charged with this task, these organizations were newly formed entities with little legitimacy in a system that was populated already by many other stakeholders. It is possible that $\mathrm{DMHs}$ were prone to choose partners that were easier to control (e.g. non-profit organizations) or that were perceived traditionally to be less powerful (e.g. social services versus health care services) and would be more likely to recognize their mandated leadership. In contrast, SAAS and DPCs are of equal organizational standing to DMHs and compete with them for funding and resources from the LHA. They can challenge the leadership role of DMHs more easily and, therefore, be engaged less convincingly in the $\mathrm{MH}$ network than the third sector and LGs.

Given the lack of evidence about the effect of different governance models, it is difficult to propose alternatives to a network leadership that is mandated by policy, such as that set up in the Italian context. On the other hand, a recent piece of work suggests that 'multiple, potentially competing managers exist in any given network, ${ }^{28}$ therefore local dynamics might be a better mechanism of selection of a network leader than policy-driven design. Alternatively, leadership could be focused within a structure that is quasi-external to the network, such as a committee or a steering group, in which governance could be shared among all stakeholders. A final approach would be to build the reputation and legitimacy of the designated network leader, the DMH. This would not be just a matter of developing the individual skills of network managers, some of which have already been discussed by numerous scholars, ${ }^{14,29,30}$ but also those network-level competencies that are needed to sustain a consensus on goals and solve conflicts among network members.

In conclusion, the findings of this study show that in promoting the creation of cross-sectoral and interorganizational collaborations, policy-makers must maintain a fine balance with respect to how much they guide the process and to what aspects they should guide. Weak and unclear incentives might leave local and professional dynamics to prevail. Rigid governance models might crystallize local relationships in suboptimal and unrealistic configurations.

The capacity of policy-makers to acknowledge and build upon the multitude of motivations that facilitate or impede the establishment of collaborations remains the ultimate challenge.

\section{Acknowledgements}

The study was supported in part by a grant from Janssen \& Cilag. We would like to thank F Longo for reviewing the manuscript critically. We thank Drs Ferrannini, Mencacci, Percudani and Pirfo, as well as all the DMH directors who participated in the survey, for their availability and enthusiasm for this topic of research.

\section{References}

1 Rochefort D. Coordination, continuity, and centralized control: a policy perspective on service strategies for the chronically mentally ill. J Soc Iss 1989;45:145-59

2 Compagni A, Adams N, Daniels A. International Pathways to Mental Health System Transformation: Strategies and Challenges. California: California Institute for Mental Health, 2007

3 Provan KG, Milward BH. Institutional-level norms and organizational involvement in a service-implementation network. J Public Admin Res Theory 1991;1:391-41

4 Provan KG, Milward $\mathrm{BH}$. A preliminary theory of interorganizational network effectiveness: a comparative study of four mental health systems. Admin Sci Quart 1995;40:1-33

5 Provan KG, Isett KR, Milward BH. Cooperation and compromise: a network response to conflicting institutional pressures in community mental health. Nonprofit Voluntary Sector Quart 2004;33:489-5146

6 Meijboom B, de Hann J, Ver heyen P. Networks for integrated care provision: an economic approach based on opportunism and trust. Health Policy 2004;69:33-43

7 Graddy EA, Chen B. Influences on the size and scope of networks for social service delivery. J Public Admin Res Theory 2006;16:533-52

8 Oliver C. Determinants of interorganizational relationships: integration and future directions. Acad Manag Rev 1990;15:241-65

9 Reitan TC. Theories of inter-organizational relations in the human services. Soc Serv Rev 1998;72:285-309

10 Provan KG, Kenis P. Modes of network governance: structure, management, and effectiveness. J Public Admin Res Theory 2008;18:229-52

11 Hultberg EL, Glendinning C, Allebeck P, Lonnroth K. Using pooled budgets to integrate health and welfare services: a comparison of experiments in England and Sweden. Health Soc Care Commun 2005;13:531-41 
12 Peck E, Gulliver P, Towell D. Modernising Partnerships - An Evaluation of Somerset's Innovations in the Commissioning and Organising of Mental Health Services. London: Institute for Applied Health and Social Policy, King's College London, 2002

13 Kenis P, Provan KG. The control of public networks. Int Public Manag J 2006;9:227-47

14 Goldsmith S, Eggers WD. Governing by Network. Washington, DC: Brookings, 2004

15 Huxham C, Vangen S. Leadership in the shaping and implementation of collaboration agendas: how things happen in a (not quite) joined-up world. Acad Manag Rev 2000;43:1159-75

16 Callaly T, Fletcher A. Providing integrated mental health services: a policy and management perspective. Aust Psychiatry 2005;13:351-6

17 Fleury MJ, Mercier C. Integrated local networks as a model for organizing mental health services. Admin Policy Mental Health Mental Health Serv Res 2002;30:55-73

18 Goldman HH, Morrissey JP, Ridgely MS. Evaluating the Robert Wood Johnson Foundation Program on chronic mental illness. Milbank Quart 1994;72:37-47

19 Morrissey PJ, Calloway M, Bartko WT, Ridgely MS, Goldman HH, Paulson IR. Local mental health authorities and service system change: evidence from the Robert Wood Johnson Program on chronic mental illness. Milbank Quart 1994;72:49-80

20 Burti L. Italian psychiatric reform 20 plus years after. Acta Psychiatr Scand Suppl 2001;104:41-6

21 de Girolamo G, Bassi M, Neri G, Ruggeri M, Santone G, Picardi A. The current state of mental health care in Italy: problems, perspectives, and lessons to learn. Eur Arch Psychiatry Clin Neurosci 2007;257:83-91

22 Van de Ven HA, Walker G, Liston J. Coordination patterns within an inter-organizational network. Hum Relations 1979;32:19-36

23 Smith Ring P, van de Ven AH. Developmental processes of cooperative inter-organizational relationships. Acad Manag Rev 1994;19:90-118

24 Isett Roussin K, Provan KG. The evolution of dyadic interorganizational relationships in a network of publicly funded nonprofit agencies. J Public Admin Res Theory 2005;15:149-65

25 Bolland JM, Wilson JV. Three faces of integrative coordination: a model of inter-organizational relations in community-based health and human services. Health Serv Res 1994;29:341-66

26 Huang K, Provan KG. Resource tangibility and patterns of interaction in a publicly funded health and human services network. J Public Admin Res Theory 2007;17:435-54

27 Mur-Veeman I, van Raak A. Integrated care: the impact of governmental behaviour on collaborative networks. Health Policy 1999;49:149-59

28 Rethemeyer RK, Hatmaker DM. Network management reconsidered: an inquiry into management of network structures in public sector service provision. J Public Admin Res Theory 2008;18:617-46

29 Bardach E. Getting Agencies to Work Together. Washington, DC: Brookings Institution, 1998

30 Alexander JA, Comfort ME, Weiner BJ, Bogue R. Leadership in collaborative community health partnerships. Nonprofit Manag Leadership 2001;12:159-75 